\title{
Avaliação da tipificação e classificação de ovos comercializados na cidade do Rio de Janeiro/RJ - Brasil
}

\section{Type and class evaluation of eggs commercialized in Rio de Janeiro/RJ - Brazil}

\author{
Lina Provenzano, ${ }^{*}$ Marta Maria Braga Xavier, ${ }^{*}$ Flávia Venturi Pimentel, ${ }^{*}$ Ismar Araújo de Moraes, ${ }^{* *}$ Gisela Cornélia Hütten, ${ }^{\star \star *}$ \\ Henrique Silva Pardi, ${ }^{* \star *}$ Sergio Borges Mano****
}

\begin{abstract}
Resumo
O objetivo do presente trabalho foi verificar a tipificação e classificação contida nos rótulos das embalagens dos ovos comercializados na cidade do Rio de Janeiro comparativamente com as normas estabelecidas na legislação vigente para este requisito. Quarenta e seis dúzias de ovos brancos e vermelhos foram avaliadas para: peso dos ovos; integridade, limpeza e deformidade da casca; altura da câmara de ar, e; ovoscopia da clara e da gema. Os ovos foram abertos com a finalidade de avaliar os resultados obtidos na ovoscopia. Realizou-se análise estatística descritiva simples, observando-se a concordância ou não com a legislação. Observou-se que 10,9\% estavam dentro dos parâmetros estabelecidos, sendo $89,1 \%$ em desacordo. Dessas (41 amostras), 51,2\% foram devido a aspectos relacionados com omissões na rotulagem; 39,0\% em relação à falhas na classificação; 39,0\% em desacordo com a tipificação apresentada na embalagem; 7,3\% com o comprometimento da integridade e limpeza da casca, e; 7,3\% apresentaram crescimento de mofo na casca. De acordo com estes resultados, concluiu-se que existe a necessidade de uma maior atenção, por parte das autoridades, na fiscalização deste produto desde a embalagem até a sua comercialização ao varejo.
\end{abstract}

Palavras-chave: ovo, qualidade.

\begin{abstract}
The objective of the present work was to verify the type and class contained in the labeling of the packaging of eggs commercialized in city of Rio de Janeiro comparatively with the norms established in the Brazilian Legislation. 46 dozens of white and red eggs had been evaluated to: eggs weight; integrity, cleanness and shell deformity; height of the air chamber, and; ovoscopy of albumen and egg yolk. Statistics was performed by simple descriptive analysis, observing it agreement or not with the current law. The results had shown that $10.9 \%$ were in agreement with legislation and $89.1 \%$ in disagreement. Of these (41 samples), $51.2 \%$ was aspects related to the omissions in labeling; $39.0 \%$ to the imperfections in the classification; $39.0 \%$ in disagreement with the presented type in the packing; $7.3 \%$ with the compromise of the integrity and cleanness of the shell, and; $7.3 \%$ had presented shell mildew formation. It can be concluded that be necessary attention by authorities in the egg's fiscalization since packing until its commercialization.
\end{abstract}

Keywords: Egg, quality.

\section{Introdução}

Segundo a Portaria no 1, de fevereiro de 1990 (Brasil, 1990), referente às Normas Gerais de Inspeção de Ovos e Derivados, entende-se pela designação "ovo", como ovo de galinha em casca, sendo os demais ovos acompanhados da indicação da espécie de que procedem.

Selecionar critérios para avaliar as mudanças na qualidade do ovo, de acordo com Rossi e Pompei (1995), implica considerar a necessidade de qualidade para produtores, consumidores e processadores. Para os produtores, a qualidade está relacionada com o peso do ovo e resistência da casca (como defeitos, sujeiras, quebras e manchas de sangue). Para os consumidores, a qualidade está relacionada com o prazo de validade do produto e com as características sensoriais, como cor da gema e da casca. Para os processadores, a qualidade está relacionada com a facilidade de retirar a casca, com a separação da gema da clara, com

* Programa de Pós-Graduação em Medicina Veterinária (Higiene Veterinária e Processamento Tecnológico de Produtos de Origem Animal) da Faculdade de Veterinária da Universidade Federal Fluminense - UFF.

** Departamento de Fisiologia e Farmacologia, Instituto Biomédico, Universidade Federal Fluminense. E-mail: fisiovet@vm.uff.br.

*** Superintendência de Controle de Zoonoses, Vigilância e Fiscalização Sanitária. Prefeitura da Cidade do Rio de Janeiro.

**** Departamento de Tecnologia dos Alimentos, Faculdade de Veterinária, Universidade Federal Fluminense. Rua Vital Brazil Filho, 64, 24230340, Niterói, RJ.

* Autor para correspondência. Sérgio Borges Mano. E-mail: mtasbm@vm.uff.br. 
as propriedades funcionais e com a cor da gema. Portanto, pode-se inferir que o controle de qualidade é um fator importante para a comercialização dos ovos, podendo ser realizado através da ovoscopia, a qual avalia a condição em que se encontra o interior do ovo. A luz do ovoscópio revela a condição da casca, o tamanho da câmara de ar, nitidez, a cor e a mobilidade da gema, bem como as condições da clara. Com esta análise, são evidenciadas anormalidades tais como mancha de sangue ou de carne, desenvolvimento embrionário e deterioração. Segundo Oliveira (2006), a ocorrência de pequenas manchas de carne ou sangue na gema ou na clara é um fator normal e não prejudica em nada o valor dos ovos, os quais podem ser consumidos normalmente. Entretanto, o desenvolvimento embrionário ou a deterioração, em hipótese alguma são aceitáveis para a comercialização (Brasil, 1997).

Para a avaliação da qualidade é analisado o aspecto externo, através do peso e da casca. O peso não é indicador de qualidade nutricional do ovo, mas serve para padronizar a comercialização. A casca é a embalagem natural dos ovos, naqueles considerados de primeira qualidade, independentemente da cor, deve ser limpa, íntegra, portanto sem sujidades, trincas e, ainda, sem deformações. Cascas resistentes ajudam a proteger a parte interna e dependem de rações com níveis suficientes e equilibrados de nutrientes como cálcio, fósforo e vitamina D3. Grandes deformações na casca prejudicam o visual e ainda indicam problemas sanitários nas poedeiras (Oliveira, 2006).

Existem cinco métodos para estimar a qualidade de ovos abertos, com bases quantitativas, relacionadas com 0 albúmen: altura da clara (Wilgus e Wagenen, 1936); índice do albúmen (Heiman e Carver, 1936); índice da área do albúmen (Parson e Mink, 1937); percentagem da clara espessa e fina (Holts e Almiquist, 1932); e a unidade "Haugh" (Haugh, 1937). O uso da unidade "Haugh" tem sido, geralmente, aceito como uma medida da qualidade do albúmen em diversas pesquisas sobre a qualidade de ovos (Eisen et al., 1962).

A unidade Haugh é uma medida norte-americana que correlaciona a altura da camada densa externa da clara com o peso do ovo. A partir destes elementos, se faz a conversão, utilizando tabela específica. A equação utilizada para calcular a unidade Haugh é: $U H=100 x \log \left(H-1,7, W^{0,37}+7,6\right)$. Outra medida de qualidade utilizada além da Unidade Haugh é o Índice da gema, sendo este calculado como a razão entre a altura e a largura da gema. Quanto maior o índice da gema, melhor a qualidade do ovo (Stadelman e Cotterill, 1994).

O objetivo do presente trabalho foi verificar a tipificação e classificação contida nos rótulos das embalagens dos ovos comercializados na cidade do Rio de Janeiro comparativamente com as normas estabelecidas na legislação vigente para este requisito.

\section{Material e métodos}

Foram utilizadas 46 dúzias de ovos brancos e vermelhos colhidos em diferentes supermercados nas áreas do Centro e Zona da Leopoldina da Cidade do Rio de Janeiro. Considerando cada dúzia como uma unidade amostral, os ovos foram coletados pelo Serviço de Vigilância Municipal e encaminhados para o Laboratório de Tecnologia dos Alimentos da Faculdade de Veterinária da Universidade
Federal Fluminense, onde foram mantidos em temperatura ambiente até o momento da análise. Os dados relativos a marca, número do órgão de fiscalização sanitária, data de produção e validade, tipo, classe e cor constantes na rotulagem foram transferidos para planilhas próprias e, a continuação, seguiu-se o protocolo de análise dos ovos.

Os equipamentos utilizados durante o procedimento de análise foram: balança analítica, utilizada para a pesagem dos ovos; ovoscópio - para visualização das câmaras de ar; paquímetro - para mensuração do diâmetro da gema; micrômetro - para verificação da altura da clara densa e da gema e para avaliar a espessura da casca. Outros equipamentos auxiliares utilizados foram: separador de gema; mesa de vidro com espelho para análise da qualidade dos ovos; escala milimétrica de $15 \mathrm{~mm}$ para medição do tamanho da câmara de ar e fichas de preenchimento de dados para o monitoramento dos mesmos.

A metodologia aplicada para a obtenção e preparo das amostras baseou-se em procedimentos de numeração e pesagem dos ovos, sendo estes pesos, em gramas, anotados em fichas para comparação posterior. Também foram anotadas nas planilhas informações como o dia de análise e o número da etiqueta lacre do órgão fiscalizador.

A análise dos ovos seguia a seguinte ordem: avaliação da integridade e limpeza da casca, altura da câmara de ar, ovoscopia de clara e gema, altura de clara densa e gema, diâmetro da gema e espessura da casca.

A avaliação das cascas foi realizada através da inspeção das mesmas onde foram anotadas na ficha correspondente alterações como cascas sujas, trincadas ou quebradas. Para mensuração do tamanho da câmara realizou-se observação individual ao ovoscópio, com demarcação da câmara de ar com auxílio de um lápis, sendo a altura medida em escala milimétrica de $15 \mathrm{~mm}$.

Posteriormente, a ovoscopia da clara e da gema foi realizada com auxílio do ovoscópio, onde se avaliou a integridade da clara e da gema e foram anotadas as alterações encontradas. Os ovos foram quebrados sobre uma mesa de vidro específica, provida de espelho na parte inferior para análise de qualidade dos ovos. A altura da clara densa e da gema foram medidas em $\mathrm{mm}$, com auxílio de um micrômetro, e o diâmetro da gema foi determinado em $\mathrm{mm}$, através da utilização do paquímetro.

A espessura da casca foi medida em milímetros $(\mathrm{mm})$, com o uso de um micrômetro, onde foi colocado um pequeno pedaço da casca para avaliação de sua espessura.

Após os procedimentos de análise dos ovos, os resultados foram tabelados e avaliados através de análise estatística descritiva simples, observando-se a concordância ou não com a legislação vigente.

\section{Resultados e discussão}

Os resultados relativos aos percentuais de amostras de ovos analisadas consideradas "em acordo" ou "em desacordo" com a legislação vigente podem ser vistos na Figura 1. Constatou-se que das 46 dúzias de ovos analisadas, 41 apresentaram fatores que não atendiam aos critérios previstos na legislação que permitissem a sua comercialização. Essas amostras em desacordo representaram $89,1 \%$ do total 
analisado, e as causas para tal classificação foram: omissão na rotulagem, classificação e tipificação inadequadas, presença de mofo e comprometimento da limpeza e integridade (Figura 2).

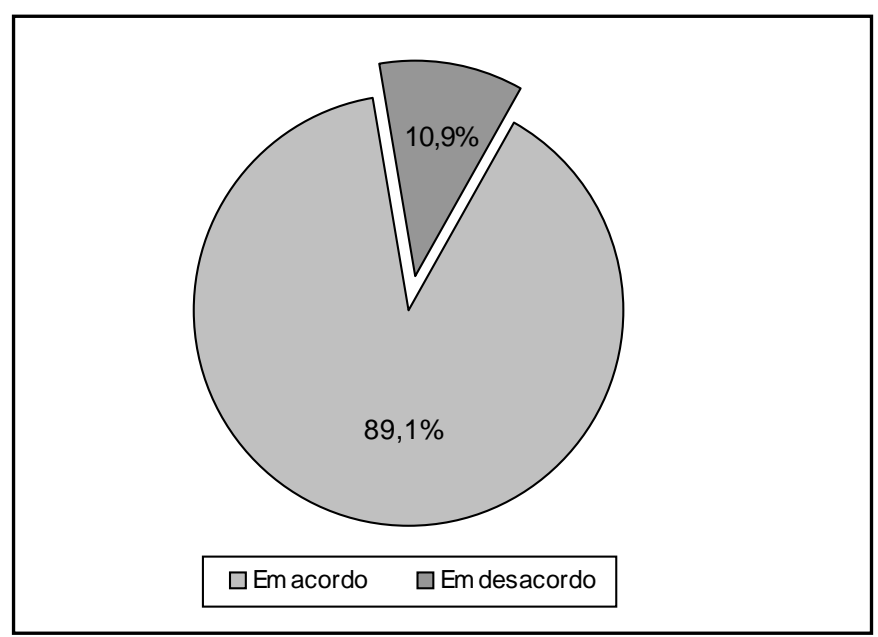

Figura 1: Percentuais de amostras de 46 dúzias de ovos analisadas na cidade do Rio de Janeiro/Brasil levando-se em consideração a rotulagem, classificação e tipificação dos ovos e posterior classificação "em acordo" ou "em desacordo" com a legislação vigente

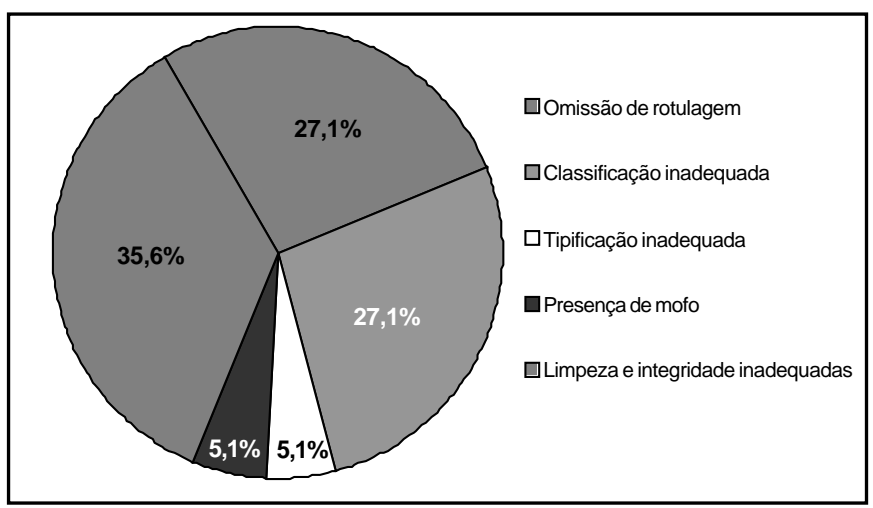

Figura 2: Percentuais de amostras de 41 dúzias de ovos julgadas em desacordo para a comercialização na cidade do Rio de Janeiro/Brasil, considerando a omissão na rotulagem, a classificação e a tipificação inadequadas, a presença de mofo e a limpeza e integridade inadequadas como fatores em desacordo com a legislação.

Dentre as amostras consideradas em desacordo, 21 apresentaram omissão de dados obrigatórios para a rotulagem, representando $51,2 \%$ do total de amostras em desacordo. Na rotulagem das embalagens devem constar dados relativos à marca, data de produção e validade, tipo, classe e cor, que têm como função orientar o distribuidor, os órgãos de vigilância e o consumidor do produto em questão. A omissão destes dados, obrigatórios na rotulagem, pode ser, em parte, justificada devido às dificuldades encontradas pelas indústrias e estabelecimentos comerciais diante da falta de orientação técnica por parte dos órgãos competentes e legisladores.
Com relação à classificação, 16 amostras apresentaram divergências entre os dados declarados e os verificados na análise. Este valor representou 39\% das amostras em desacordo. De acordo com a Resolução CIPOA n 005 de 19 de novembro de 1991 (Brasil, 1991), os ovos são ordenados em 5 classes, que são: A, B, C, D e E, onde características da casca (limpeza, integridade, manchas e deformações), da câmara de ar (tamanho e localização), do albúmen (limpidez, transparência, consistência e integridade das calazas) e da gema (localização, forma, consistência e desenvolvimento de germe) são avaliados e classificados de acordo com a qualidade.

Também foram observadas divergências entre a tipificação declarada e a analisada em 16 amostras, representando 39\% das dúzias em desacordo. Segundo seu peso, os ovos podem ser divididos em 6 tipos, de acordo com a legislação (Brasil, 1991): Tipo 1 (jumbo) - peso mínimo acima de 66g/unidade; Tipo 2 (extra) - peso entre 60-65g/unidade; Tipo 3 (grande) peso mínimo entre 55-60g/unidade; Tipo 4 (médio) - peso mínimo entre 50-55g/unidade; Tipo 5 (pequeno) - peso mínimo entre 45-50g/unidade; Tipo 6 (industrial) - peso abaixo de $45 \mathrm{~g} /$ unidade.

Os problemas encontrados na tipificação e classificação, provavelmente, deveram-se à falta de uma cadeia de frio durante os períodos de transporte e armazenamento, com o conseqüente comprometimento da qualidade do produto final, sendo refletido nos parâmetros de tipificação e classificação, fato este comprovado por Kraemer et al. (2003). Deve-se também aventarse a possibilidade de erros na classificação e tipificação desde a sua origem na granja ou entreposto, problema este devido à falta de assistência técnica e fiscalização por parte dos órgãos competentes para tal finalidade.

No que se refere à presença de mofo nas cascas dos ovos analisados, foram observadas três amostras comprometidas, representando $7,3 \%$ do total de amostras em desacordo. Com relação ao comprometimento da integridade e limpeza da casca, foram observadas três amostras em desacordo, representando $7,3 \%$ do total das amostras em desacordo. Os ovos destinados ao consumo in natura devem apresentar casca limpa e íntegra, admitindo-se defeitos de textura, contorno e manchas, estes últimos podem interferir na classificação final do produto. O aparecimento de mofo, bem como o comprometimento da limpeza e integridade são decorrentes de falhas na manipulação, seja na produção ou na comercialização. Estes fatores diminuem a qualidade final do produto e a aceitação por parte do consumidor. Uma alternativa para contornar este problema é a implementação do sistema de Boas Práticas, através do treinamento e conscientização de manipuladores a fim de diminuir a incidência deste tipo de desacordo.

\section{Conclusão}

Os resultados obtidos permitem concluir que a maior parte das marcas de ovos comercializadas na cidade do Rio de Janeiro, não está dentro das normas legais, sendo principalmente observadas falhas na rotulagem e nas características de tipificação e classificação, devendo os órgãos federais, estaduais e municipais envolvidos com a vigilância e fiscalização adotarem os procedimentos 
necessários, com o propósito de garantir a exposição e a venda de produtos saudáveis, de qualidade e dentro dos

\section{Referências}

BRASIL. Portaria oㅡ 01, de 21 de fevereiro de 1990. Ministério da Agricultura e do Abastecimento. Secretaria de Defesa Agropecuária. Divisão de Inspeção de Carnes e Derivados - DICAR. Normas Gerais de Inspeção de Ovos e Derivados. Diário Oficial da República Federativa do Brasil, Brasília, DF, n. 44, p. 4321, 6 de mar. 1990. Seção 1.

BRASIL. Ministério da Agricultura e do Abastecimento. Secretaria de Defesa Agropecuária. Divisão de Inspeção de Carnes e Derivados DICAR. Resolução 005 de 19/11/91 da Coordenação de Inspeção de Produtos de Origem Animal-CIPOA. 1991.

BRASIL. Ministério da Agricultura e do Abastecimento. Secretaria de Defesa Agropecuária. Departamento de Inspeção de Produtos de Origem Animal. Regulamento da Inspeção Industrial e Sanitária de produtos de Origem Animal (RIISPOA). Aprovado pelo Decreto ํㅜ 30.691, 29/03/52, alterado pelos Decretos no 1255 de 25/06/62, 1236 de 02/ 09/94, 1812 de 08/02/96 e 2244 de 04/06/97, Brasília, DF, 1997, 241 p.

EISEN, E.J.; BOHRE, B.B.; MCKEAN, H.E. The Haugh unit as a measure of egg albumen quality. Poultry Science, v. 41, p. 1461-1468, 1962.

HAUGH, R.R. The Haugh unit for measuring egg quality. United States Egg Poultry Magazine, v. 43, p. 552-555, 1937.

HEIMAN, V.; CARVER, J.S. The albumen index as a physical measurement of observed egg quality. Poultry Science, v. 15, p. 141$148,1936$. padrões legais, conforme previsto no Código de Defesa do Consumidor.

HOLTS, W.F.; ALMIQUIST, H.J. Measurement of deterioration in the stored hen's egg. United States Egg Poultry Magazine, v. 38, p. 70, 1932.

KRAEMER, F.B.; HÜTTEN, G.C.; TEIXEIRA, C.E.;PARDI, H.S.; MANO, S. Avaliação da qualidade interna de ovos em função da variação da temperatura de armazenamento. Revista Brasileira de Ciência Veterinária, v.10, n. 3 p. 145-151, 2003.

OLIVEIRA, B. L. Ovo - Qualidade e importante. [online] Disponível: http:// www.snagricultura.org.br/artigos/artitec-ovos.htm. Arquivo capturado em 30 de junho de 2006.

PARSONS, C.H.; MINK, L.D. Correlation of methods for measuring the interior quality of eggs. United States Egg Poultry Magazine, v. 43, p. 484-489, 1937.

ROSSI, M.; POMPEI, C. Changes in some egg components and analytical values due to hen age. Poultry Science, v. 74, p. 152-160, 1995.

STADELMAN, W.J., COTTERILL, OWEN J. (ed.). Egg Science and Technology. 4. ed. New York: The Haworth Press, 1994. 591 p.

WATKINS, B.A. The nutritive value of the egg. In: STADELMAN, W.J. \& COTTEILL, O.J. Egg Science and Technology. 4. ed. Binghamton, New York, 1994. 591 p. cap. 7, p. 177-194.

WILGUS, H.S.; WAGENEN, A. The heigh of the firm albumen as a measure of its condition. Poutry Science, v. 15, p. 319-321, 1936. 